\title{
Long-Term Oxygen Therapy and Risk of Fire-Related Events
}

\author{
Conner Moslander, DO; Tasnim Lat, DO; Badri Giri, MD; Rachael Pattison; John D. Coppin, MPH; \\ and Udaya M. Bhat, MD
}

Introduction: Two large major trials showed that long-term oxygen therapy (LTOT) improved mortality in patients with chronic obstructive pulmonary disease (COPD) and hypoxemia. Although oxygen accelerates combustion and is an obvious fire hazard, LTOT has traditionally been prescribed to veterans who are actively smoking.

Methods: We conducted a retrospective chart review of all veterans with COPD at a single center who were prescribed new LTOT between October 2010 and September 2015. Of the 158 patients who met the study criteria, 152 were male. Bayesian logistic regression was used to model the outcome variable fire-related incident with the predictors smoking status, age, race, depression, posttraumatic stress disorder, and type of oxygen used.

Results: The mean age of the 158 patients with COPD in the study was 71.3 years in nonsmokers and 65.9 years in smokers. The model-estimated odds (SD) of a fire-related incident occurring in a smoker were 31.6 (5.1-372.7) times the odds of a fire-related incident occurring in a nonsmoker.

Conclusions: Patients who smoke and remain on LTOT put themselves at greater risk of having a fire-related incident than do nonsmokers.
Author affiliations can be found at the end of the article.

Correspondence: Udaya Bhat

(udaya.bhat@va.gov)

Fed Pract. 2020;37(10):442-446. doi: $10.12788 /$ fp. 0048
? hronic obstructive pulmonary disease (COPD) has been the third leading cause of death in the US since 2008. ${ }^{1}$ Current management of COPD includes smoking cessation, adequate nutrition, medication therapy, pulmonary rehabilitation, and vaccines. ${ }^{2}$ Outside of pharmacologic management, oxygen therapy has become a staple treatment of chronic hypoxemic respiratory failure due to COPD. Landmark trials, including the Nocturnal Oxygen Therapy Trial (NOTT) and Medical Research Council (MRC) study, demonstrated improved survival in patients with COPD and hypoxemia, particularly if these patients received oxygen for 18 hours per day. ${ }^{3,4}$ NOTT prospectively evaluated 203 patients at 6 centers who were randomly allocated to either continuous oxygen therapy or 12-hour nocturnal oxygen therapy. The overall mortality in the nocturnal oxygen therapy group was 1.94 times that in the continuous oxygen therapy group $(P=.01){ }^{3}$ The MRC study included 87 patients who were randomized to oxygen therapy or no oxygen; risk of death was $12 \%$ per year in the treated group vs $29 \%$ per year in the control group $(P=.04) .^{4}$ The effectiveness of long-term oxygen therapy (LTOT) in active smokers continues to be a source of debate; although $50 \%$ of patients in the NOTT trial were smokers, there was no subgroup analysis of whether smoking status had an impact on survival in those on continuous oxygen therapy.

Although many therapies are available for the treatment of COPD, the most effective treatment to prevent the progression of COPD is smoking cessation. Resources like smoking cessation programs, nicotine patches, and medications, such as bupropion and varenicline, are available to aid smoking cessation. ${ }^{5}$ However, many patients are unable to quit tobacco use despite their best efforts using available resources, and they continue to smoke even with progressive COPD. Long-time smokers also are likely to continue smoking while on LTOT, which increases their risk for fire-related injury. ${ }^{6-8}$

Traditional indications are being scrutinized after the LTOT trial found no benefit with respect to time to death or first hospitalization among patients with stable COPD and resting or exercise-induced moderate desaturation. ${ }^{9}$

Although oxygen accelerates combustion and is a potential fire hazard, LTOT has been prescribed even to active smokers as the 2 landmark trials did not exclude patients who were active smokers from receiving oxygen therapy. ${ }^{3,4}$ Therefore, LTOT has traditionally been prescribed to veterans who are actively smoking, despite the fire hazard. Attempts at mitigating hazards related to oxygen therapy in active smokers include counseling extensively about safety measures (which includes avoiding open flames such as candles, large fires, or sparks 
TABLE 1 Baseline Characteristics Nonsmokers vs Smokers

\begin{tabular}{lcc} 
Characteristics & Nonsmokers $(\mathbf{n}=\mathbf{1 0 5})$ & Smokers $(\mathbf{n}=\mathbf{5 3})$ \\
\hline $\begin{array}{l}\text { Demographics } \\
\text { Age, mean, median (SD), y }\end{array}$ & $71.3,70(8.5)$ & $65.9,66(6.6)$ \\
Non-White, No. (\%) & $15(14.3)$ & $6(11.3)$ \\
\hline Conditions, No. (\%) & & \\
$\quad$ Fire-related incident & $0(0)$ & $9(17.0)$ \\
Depression & $17(16.2)$ & $17(32.1)$ \\
Posttraumatic stress disorder & $8(7.6)$ & $6(11.3)$ \\
Continuous oxygen & $36(34.3)$ & $22(41.5)$
\end{tabular}

when on LTOT and providing Home Safety Agreements-a written contract between prescriber and patient wherein the patient agrees to abide by the terms of the US Department of Veterans Affairs (VA) to mitigate hazards related to LTOT in order to receive LTOT (eAppendix can be found at doi:10.12788/fp.0048). These clinical techniques ensure that patients who choose to smoke on LTOT do so only with a full understanding of the dangers.

\section{METHODS}

With this practice in mind, we conducted an institutional review board approved retrospective chart review of all veterans with diagnosis of COPD within the Central Texas Veterans Health Care System (CTVHCS) who were prescribed new LTOT between October 1, 2010 and September 30, 2015. Given the retrospective nature of the chart review, patient consent was not obtained. Inclusion criteria were veterans aged $>18$ years who had a confirmed diagnosis of COPD by spirometry and who met criteria for either continuous or ambulation-only oxygen therapy.

Criteria for exclusion included patients with hypoxemia not solely attributable to COPD or due to diseases other than COPD. We reviewed encounters in these patients' charts, including follow-up in the clinic of the providers prescribing oxygen, to assess for fire-related incidents, defined as events wherein fire was visualized by the patient or by individuals living with the patient and with report provided to medical equipment company providing oxygen; the patient did not have to seek medical care to qualify for fire-related incident. Of the 158 patients who met the criteria for inclusion in the study, 152 were male.

\section{Statistics}

Bayesian logistic regression was used to model the outcome variable fire-related incident with the predictors smoking status, age, race, depression, PTSD, and type of oxygen used. Mental health disorders have significant effect on substance use disorders, such as alcohol use. Depression and PTSD were more common mental health diagnoses found in our patient population. Additionally, due to the small sample size, these psychiatric diagnoses were chosen to evaluate the impact of mental health disorders on firerelated events.

Although the sample size of events was small, weakly informative normal priors $(0,2.5)$ were used to shrink parameter estimates toward 0 and minimize overfitting. Weakly informative normal priors have also been suggested to deal with the problem of quasi-complete separation, where in our case, both smoking and no-PTSD perfectly predicted the 9 fire-related incidents. ${ }^{10}$ All input variables were centered and scaled as recommended. ${ }^{9}$ The model fit well as assessed by posterior predictive checks, and Rhat was 1.00 for all parameters, indicating that all chains converged. Analysis was completed in $\mathrm{R}$ version 3.5.1 using the 'brms' package for Bayesian modeling. ${ }^{11}$

\section{RESULTS}

The mean age for the 158 included patients was 71.3 years in nonsmokers and 65.9 years in smokers. Fifty-three of the included patients were active smokers when LTOT was initiated. Nine veterans had fire-related incidents during the study period. All 9 patients were actively smoking (about 17\%) at the time of the fire incidents. There were no deaths, and 5 patients required hospitalization due to facial burns resulting from the 
TABLE 2 Bayesian Logistic Regression Model

\begin{tabular}{lccc} 
Characteristics & Coefficient & Estimated Error (coefficient) & Odds Ratio (95\% Cl) \\
\hline Smoking & 3.53 & 1.08 & $31.6(5.1-372.7)$ \\
\hline Age & 0.18 & 0.85 & $1.2(0.2-6.5)$ \\
\hline White & -0.22 & 1.12 & $0.9(0.1-6.1)$ \\
\hline Depression & 0.37 & 0.85 & $1.5(0.2-7.3)$ \\
\hline Posttraumatic stress disorder & -2.13 & 1.58 & $0.1(0.0-1.8)$ \\
\hline Type oxygen, continuous & 0.10 & 0.74 & $1.1(0.3-4.8)$ \\
\hline Male & -1.66 & 1.82 & $0.2(0.0-4.3)$
\end{tabular}

${ }^{a}$ Median odds ratio and 0.025 and 0.975 quantile of the posterior distribution.

fire-related incidents. Our study focused on 5 baseline characteristics in our population (Table 1). After gathering data, our group inferred that these characteristics had a potential relationship to fire-related incidents compared with other variables that were studied. Future studies could look at other patient characteristics that may be linked to fire-related incidents in patients on LTOT. For example, not having PTSD also perfectly predicts fire-related incidents in our data (ie, none of the participants who had fire-related incidents had PTSD). Although this finding was not within the $95 \%$ confidence interval $(\mathrm{CI})$ in the model, it does show that care must be taken when interpreting effects from small samples (Table 2). The modelestimated odds of a fire-related incident occurring in a smoker were $31.6(5.1-372.7)$ times more likely than were the odds of a firerelated incident occurring in a nonsmoker, holding all other predictors at their reference level; 95\% CI for the odds ratios for all other predictors in the model included a value of 1 .

\section{DISCUSSION}

This study showed evidence of increased odds of fire-related events in actively smoking patients receiving LTOT compared with patients who do not actively smoke while attempting to adjust for potential confounders. Of the 9 patients who had fire events, 5 required hospitalization for burns.

A similar retrospective cohort study by Sharma and colleagues in 2015 demonstrated an increased risk of burn-related injury when on LTOT but reiterated that the benefit of ox- ygen outweighs the risk of burn-related injury in patients requiring oxygen therapy. ${ }^{12}$ Interestingly, Sharma and colleagues were unable to identify smoking status for the patients studied but further identified factors associated with burn injury to include male sex, low socioeconomic status, oxygen therapy use, and $\geq 3$ comorbidities. The study's conclusion recommended continued education by health care professionals (HCPs) to their patients on LTOT regarding potential for burn injury. In the same vein, the VA National Center for Ethics in Health Care noted that "clinicians should familiarize themselves with the risks and benefits of LTOT; should inform their patients of the risks and benefits without exaggerating the risk associated with smoking; avoid undue coercion inherent in the clinician's ability to withdraw LTOT; reduce the risk to the greatest degree possible; and consider termination of LTOT in very extreme cases and in consultation with a multidisciplinary committee."13

This statement is in contrast to the guidelines and policies of other countries, such as Sweden, where smoking is a direct contraindication for prescription of oxygen therapy, or in Australia and New Zealand, where the Thoracic Society of Australia and New Zealand oxygen therapy guidelines recommend against prescription of LTOT, citing "increased fire risk and the probability that the poorer prognosis conferred by smoking will offset treatment benefit." ${ }^{6,14}$

The prevalence of oxygen therapy introduces the potential for fire-related incidents with subsequent injury requiring 
medical care. There are few studies regarding home oxygen fire in the US due to the lack of a uniform reporting system. One study by Wendling and Pelletier analyzed deaths in Maine, Massachusetts, New Hampshire, and Oklahoma between 2000 and 2007 and found 38 deaths directly attributable to home oxygen fires as a result of smoking. ${ }^{15}$ Further, the Consumer Product Safety Commission's National Electronic Injury Surveillance System between 2003 and 2006 attributed 1,190 thermal burns related to home oxygen fires; the majority of which were ignited by tobacco smoking. ${ }^{15}$ The Swedish National Register of Respiratory Failure (Swedevox) published prospective population-based, consecutive cohort study that collected data over 17 years and evaluated the risk of fire-related incident in those on LTOT. Of the 12,497 patients sampled, 17 had a burn injury and 2 patients died. The low incidence of burn injury on LTOT was attributed to the strict guidelines instituted in Sweden for doctors to avoid prescribing LTOT to actively smoking patients. ${ }^{6} \mathrm{~A}$ follow-up study by Tanash and colleagues compared the risk of burn injury in each country, respectively. The results found an increased number of burn injuries in those on oxygen therapy in Denmark, a country with fewer restrictions on smoking compared with those of Sweden. ${ }^{7}$ Similarly, our results showed that the rate of fire and burn injuries was exclusively among veterans who were active smokers. All patients who were prescribed oxygen therapy at CTVHCS received counseling and signed Home Safety Agreements. Despite following the recommendations set forth by the VA on counseling, extensive harm reduction techniques, and close follow-up, we found there was still a high incidence of fires in veterans with COPD on LTOT who continue to smoke.

The findings from our study concur with those previously published regarding the risk of home oxygen fire and concomitant smoking, supporting the idea for more regulated and concrete guidelines for prescribing LTOT to those requiring it. ${ }^{8}$

\section{LIMITATIONS}

The major limitation was the small sample size of our study. Another limitation was that our study population is predominantly male as is common in veteran cohorts. In fiscal year 2016, the veteran population of Texas was $1,434,361$ males and 168,967 females. ${ }^{16}$ According to Franklin and colleagues, HCPs noticed an increase use of long-term oxygen among women compared with that of men. ${ }^{17}$

\section{CONCLUSIONS}

Our study showed an increased odds of firerelated incidents of patients while on LTOT, strengthening the argument that even with extensive education, those who smoke and are on LTOT continue to put themselves at risk of a fire-related incident. This finding stresses the importance of continuing patient education on the importance of smoking cessation prior to administration of LTOT or avoiding fire hazards while on LTOT. Further research into LTOT and fire hazards could help in implementing a more structured approval process for patients who want to obtain LTOT. We propose further studies evaluating risk factors for the incidence of fire events among patients prescribed LTOT. A growing and aging population with a need for LTOT necessitates examination of oxygen safe prescribing.

\section{Author affiliations \\ Conner Moslander is a Resident in the Department of Internal Medicine; Tasnim Lat is Faculty and Rachael Pattison is a Fellow, both in the Division of Pulmonary/Critical Care Medi- cine; all at Baylor Scott \& White in Temple, Texas. Badri Giri is an Assistant Professor at Virginia Tech Carilion School of Medi- cine in the Pulmonary, Critical Care and Sleep Medicine Car- ilion Clinic in Roanoke, Virginia. John Coppin is a Statistician in the Department of Research, and Udaya Bhat is Associate Program Director for the Pulmonary and Critical Care Fellow- ship Program, both at Central Texas Veterans Health Care System. Udaya Bhat is Chief, Pulmonary/Critical Care Section and Assistant Professor of Medicine at Texas A\&M University in College Station.}

\section{Acknowledgments}

We thank Central Texas Veterans Health Care System for its support in this project.

\section{Author disclosures}

The authors report no actual or potential conflicts of interest with regard to this article.

\section{Disclaimer}

The opinions expressed herein are those of the authors and do not necessarily reflect those of Federal Practitioner, Frontline Medical Communications Inc., the US Government, or any of its agencies.

\section{References}

1. $\mathrm{Ni} \mathrm{H}, \mathrm{Xu}$ J. COPD-related mortality by sex and race among adults aged 25 and over: United States 2000-2014. https:// www.cdc.gov/nchs/data/databriefs/db256.pdf. Published 
September 2016. Accessed September 10, 2020.

2. Itoh M, Tsuji T, Nemoto K, Nakamura H, Aoshiba K. Undernutrition in patients with COPD and its treatment. Nutrients. 2013;5(4):1316-1335. doi:10.3390/nu5041316

3. Continuous or nocturnal oxygen therapy in hypoxemic chronic obstructive lung disease: a clinical trial. Nocturnal Oxygen Therapy Trial Group. Ann Intern Med. 1980;93(3):391. doi:10.7326/0003-4819-93-3-391

4. Long term domiciliary oxygen therapy in chronic hypoxic cor pulmonale complicating chronic bronchitis and emphysema. Report of the Medical Research Council Working Party. Lancet. 1981;1(8222):681-686. doi:10.1016/S0140-6736(81)91970-X

5. Anthonisen NR, Skeans MA, Wise RA, Manfreda J, Kanner RE, Connett JE. The effects of a smoking cessation intervention on 14.5-year mortality. Ann Intern Med. 2005;142(4):233-239. doi:10.7326/0003-4819-142-4 $-200502150-00005$

6. Tanash HA, Huss F, Ekström M. The risk of burn injury during long-term oxygen therapy: a 17-year longitudinal national study in Sweden. Int J Chron Obstruct Pulmon Dis. 2015;10:2479-2484. doi:10.2147/COPD.S91508

7. Tanash HA, Ringbaek T, Huss F, Ekström M. Burn injury during long-term oxygen therapy in Denmark and Sweden: the potential role of smoking. Int J Chronic Obstruct Pulmon Dis. 2017;12:193-197. doi:10.2147/COPD.S119949

8. Kassis SA, Savetamal A, Assi R, et al. Characteristics of patients with injury secondary to smoking on home oxygen therapy transferred intubated to a burn center. J Am Coll Surg. 2014;218(6):1182-1186. doi:10.1016/j.jamcollsurg.2013.12.055

9. Long-Term Oxygen Treatment Trial Research Group, Albert $\mathrm{RK}, \mathrm{Au} \mathrm{DH}$, et al. A Randomized Trial of Long-Term Oxy gen for COPD with Moderate Desaturation. N Engl J Med. 2016;375(17):1617-1627. doi:10.1056/NEJMoa1604344

10. Ghosh J, Li Y, Mitra R. On the use of Cauchy prior distri- butions for Bayesian logistic regression. Bayesian Anal. 2018;13(2):359-383. doi:10.1214/17-ba1051

11. Bürkner P-C. brms: An R package for Bayesian multilevel models using Stan. J Stat Software. 2017;80(1). doi:10.18637/jss.v080.i01

12. Sharma G, Meena R, Goodwin JS, Zhang W, Kuo Y-F, Duarte AG. Burn injury associated with home oxygen use in patients with chronic obstructive pulmonary disease. Mayo Clin Proc. 2015;90(4):492-499. doi:10.1016/j.mayocp.2014.12.024

13. US Department of Veterans Affairs, National Ethics Committee. Ethical considerations that arise when a home care patient on long term oxygen therapy continues to smoke. http://vaww.ethics.va.gov/docs/necrpts /NEC Report 20100301 Smoking while on LTOT.pdf. Published March 2010. [Nonpublic, source not verified.]

14. McDonald C F, Whyte K, Jenkins S, Serginson J. Frith $P$. Clinical practice guideline on adult domiciliary oxygen therapy: executive summary from the Thoracic Society of Australia and New Zealand. Respirology. 2016;21(1):76-78. doi:10.1111/resp.12678

15. Centers for Disease Control and Prevention (CDC). Fatal fires associated with smoking during long-term oxygen therapy--Maine, Massachusetts, New Hampshire, and Oklahoma, 2000-2007. MMWR Morb Mortal Wkly Rep. 2008;57(31):852-854.

16. US Department of Veteran Affairs. National Center for Veterans Analysis and Statistics. Population tables: the state, age/gender, 2016. https://www.va.gov/vetdata/Veteran_Population.asp. Updated August 5, 2020. Accessed September 11, 2020.

17. Franklin KA, Gustafson T, Ranstam J, Ström K. Survival and future need of long-term oxygen therapy for chronic obstructive pulmonary disease--gender differences. Respir Med. 2007;101(7):1506-1511. doi:10.1016/j.rmed.2007.01.009 